\title{
Teletrabajo en pandemia: la crisis que le dio a la comunicación interna su gran oportunidad
}

\section{Teleworking during the pandemic: the crisis that gave internal communication its biggest chance}

\author{
Dra. Mónica Arzuaga-Williams \\ Universidad Católica del Uruguay. Uruguay. \\ marzuaga@ucu.edu.uy \\ https://orcid.org/0000-0002-1193-287X \\ Mgter. Ana Inés Pepe Arias \\ Universidad Católica del Uruguay. Uruguay. \\ anpepe@ucu.edu.uy \\ https://orcid.org/0000-0003-1704-4088 \\ Mgter. Adolfo Umpiérrez Echenique \\ Universidad Católica del Uruguay. Uruguay. \\ Adolfo.umpierrez@ucu.edu.uy \\ https://orcid.org/0000-0002-5088-5244 \\ Recepción: 17/11/2021 Revisión: 16/12/2021 \\ Aceptación: 18/12/2021 Publicación: 28/12/2021
}

\begin{abstract}
Resumen
El artículo presenta un estudio sobre los desafíos que la pandemia de COVID-19 tuvo para los profesionales de la comunicación interna en Uruguay. Para ello, se realizaron 15 entrevistas en profundidad con responsables de la comunicación interna de grandes empresas privadas, durante los meses de abril y mayo de 2021. Durante la crisis generada por la pandemia los profesionales lograron importantes aprendizajes sobre los impactos de pasar al teletrabajo de forma abrupta, en cuanto a la necesidad de fortalecer los valores de cercanía y confianza, y el papel de los líderes como agentes de comunicación. Se concluye que para superar este desafío las unidades de comunicación interna implementaron nuevos canales y prácticas, los profesionales desarrollaron aún más el rol de asesoría interna y lograron un reconocimiento amplio y positivo como función estratégica. No solo se identifican claramente las oportunidades del teletrabajo, sino también sus riesgos, especialmente para la motivación y el bienestar de los empleados, que se han configurado como prioridades del área en este contexto.
\end{abstract}

\section{Abstract}

The article describes a study regarding the challenges that the COVID-19 pandemic posed to internal communication professionals in Uruguay. For this, 15 in-depth interviews to people who are responsible for the internal communication of large private companies were conducted during the months of April and May 2021. During the crisis generated by the pandemic, the professionals acquired significant knowledge concerning the impacts of moving to telework abruptly, the need to strengthen the values of proximity and trust, and the role of leaders as communication agents. It is concluded that to overcome this challenge the internal communication units implemented new channels and practices, the professionals developed the role of internal advisory further, and they achieved wide recognition regarding their strategic function. Not only are the opportunities of teleworking clearly identified, but also its risks, especially for the motivations and well-being of employees, which have been configured as priorities of the area in this context.

Palabras clave: Comunicación interna; COVID-19; Teletrabajo; Uruguay.

Keywords: Internal communication; COVID-19; Telework; Uruguay. 


\section{Introducción}

La generalización del trabajo remoto provocada por las medidas tomadas por gobiernos y empleadores durante la pandemia del COVID-19, deja en evidencia algo afirmado desde hace tiempo: las organizaciones no están constituidas por el espacio físico que habitan, sino por la comunicación (Putnam y Nicotera, 2009). Para que las empresas pudieran cumplir su misión y sigan operativas durante las medidas que impuso el trabajo remoto en los distintos países, fue necesario mantener en marcha las conversaciones entre los integrantes, aun cuando ya no estaban físicamente en sus lugares de trabajo. Esa función quedó en el ámbito de los departamentos dedicados a la comunicación organizacional interna.

La comunicación interna es una especialización dentro de las relaciones públicas o la comunicación organizacional, corporativa o institucional (las denominaciones habituales cambian de acuerdo con el país y el tipo de organización) que ha estado en crecimiento desde los años 90 del siglo pasado (Verčič, et al., 2012). La globalización, las crisis económicas, las metodologías de trabajo como la calidad total o la reingeniería y por supuesto, la incorporación de tecnología, generan una serie de cambios en las empresas que se complejizan y profundizan, y que hacen más necesaria la gestión de los comportamientos, actitudes y emociones de los empleados y también más desafiante su satisfacción y retención, por lo que la comunicación interna se vuelve imprescindible para el desarrollo organizacional.

Pasando el 2000 empiezan a establecerse institutos y asociaciones específicas de profesionales de comunicación interna tanto en Europa como en América. A modo de ejemplo, la Asociación Argentina de Comunicación Interna fue creada en 2007 y es una de las primeras a nivel mundial y latinoamericano, en 2010 se forma el Instituto de Comunicación Interna de Gran Bretaña, y en 2014 la Asociación Uruguaya de Comunicación Interna.

Esta mayor profesionalización del área no necesariamente se reflejaba antes de la pandemia en una mayor priorización de la función en las organizaciones. En una encuesta realizada en 2019 a 215 profesionales de la comunicación organizacional en Uruguay, solamente uno de cada cuatro profesionales de este campo indicaba que su organización priorizaba la comunicación interna, fundamentalmente para informar a los integrantes de la organización sobre asuntos de trabajo, tareas, proyectos y resultados de la organización (Arzuaga-Williams, 2020).

Por esto resulta significativa la diferencia con los resultados de la edición 2020-2021 del Latin American Communication Monitor (Álvarez-Nobell, A. et al., 2021) en la que se investigó, entre otros temas, la percepción de los profesionales de la comunicación de toda Latinoamérica sobre el impacto que tuvo el COVID-19 en sus organizaciones. Allí la misma investigadora en conjunto con otras colegas realizaron el reprocesamiento y análisis de los resultados de la situación en Uruguay que fue presentado el 30 de septiembre de 2021 (Arzuaga-Williams et al., 2021). De este reporte surge que para el $92 \%$ de los profesionales uruguayos la función de la comunicación tuvo importancia alta o muy alta para atender los impactos de la pandemia, y el $52 \%$ de los comunicadores afirmó que sus empresas priorizaron durante la pandemia la relación con los públicos internos, sus empleados o colaboradores. Este reposicionamiento de la función de la comunicación interna indudablemente fue impulsado por las medidas restrictivas de la movilidad adoptadas por los gobiernos durante la pandemia, que obligaron a pasar al teletrabajo en todos los casos posibles.

Específicamente en Uruguay el 13 de marzo de 2020, con cuatro casos confirmados, la presidencia anuncia la emergencia sanitaria con medidas de cierre de fronteras, la exhortación a realizar el trabajo a distancia, el uso del tapabocas, y otras medidas de higiene. Las medidas se ajustaron en el correr de la pandemia con requisitos de aforos 
y el cierre de actividades que implican grandes concentraciones de personas, pero en ningún momento se impuso cuarentena o aislamiento forzoso, salvo para las personas diagnosticadas o en contacto con enfermos por COVID-19.

En Uruguay la ley 19.978, de "Promoción y Regulación del Teletrabajo" fue promulgada por el Poder Ejecutivo a mediados de agosto de 2021, cuando la emergencia sanitaria se encontraba en relativo control. Entre sus artículos, define el teletrabajo como un acuerdo voluntario y reversible y asegura al teletrabajador la igualdad de derechos y condiciones con el resto de los trabajadores. Entre otros factores, regula el derecho a la desconexión, la limitación de la jornada laboral y la provisión de los elementos necesarios para el teletrabajo por parte del empleador.

Aunque en Uruguay el teletrabajo ya funcionaba para algunas empresas y algunas personas, los teletrabajadores en 2019 eran solamente un 6\% del total de las personas que se conectaron a internet (Agencia de Gobierno Electrónico y Sociedad de la Información y del Conocimiento, 2020). La pandemia impulsó la adopción abrupta de esta modalidad, en un contexto particular, ya que la situación sanitaria y económica ponía en riesgo el empleo y el bienestar físico y mental de los trabajadores de la organización.

El teletrabajo es una alternativa valorada y puesta en práctica por algunos empresarios para impulsar el desempeño en las organizaciones que lideran (Morelos-Gómez \& LoraGuzmán, 2021). Esto sumado a que el teletrabajo se presenta como una oportunidad para descentralizar la actividad de las empresas e impulsar la competencia en aquellas organizaciones con intenciones de convertirse en esas empresas digitales a las que siempre aspiraron (Vattuone, 2021). Pero algunos autores sostienen que, teniendo en cuenta que a partir de la pandemia el teletrabajo llegó para quedarse, representa riesgos para los trabajadores por la invisibilidad del trabajo colectivo que lleva a reforzar el individualismo y la flexibilidad que expone al trabajador al abuso laboral (Vattuone, 2021).

Este fenómeno desencadenó en organizaciones de todo el mundo un aumento de las comunicaciones a través de los canales digitales que ya se utilizaban y la exploración, desarrollo y uso de nuevas plataformas para la comunicación interna (Xifra, 2020). Todo esto en un contexto particular de crisis.

En tiempos de crisis el cambio que se da en la organización y, por lo tanto, en la gestión de la comunicación dependen, en gran medida, de la capacidad de cambiar el estilo de comunicación de quien ejerce liderazgo (Bojadjiev y Vaneva, 2021). Los líderes de las organizaciones juegan un papel muy importante durante la experiencia que significó el teletrabajo forzado. Para muchas empresas esto define la importancia de identificar indicadores para reformular los criterios de selección y capacitación de quienes ejercerán el liderazgo dentro de las empresas, teniendo en cuenta que ese liderazgo sería virtual. Estudios realizados durante la pandemia muestran que aquellos empleados que accedieron a una comunicación de crisis de alta calidad por parte de sus líderes mostraron menos incertidumbre relacionada al impacto de la crisis ocasionada por la pandemia de COVID-19 y esto se vio acompañado por un menor agotamiento emocional (Dolce et al., 2020).

La distancia del teletrabajo pone a las empresas en la necesidad de distribuir el poder involucrando a los empleados en ciertos procesos de toma de decisiones. Esto requiere que los líderes analicen y prioricen la información importante, en medio de una marea de datos disponibles, para responder de manera más rápida e innovadora, y favorecer la toma de decisiones descentralizada (Dolce, et, al. 2020). De esta manera, el rol de liderazgo se vuelve sustancial para alcanzar los resultados organizacionales y el bienestar de los trabajadores cuando se trata de una crisis y en especial en el trabajo remoto (Contreras, et al, 2020). 
La influencia de los líderes formales es tanto o más relevante en el teletrabajo que en la modalidad tradicional. Los líderes son quienes deben trabajar con sus colaboradores para construir equipos virtuales efectivos y funcionales para alcanzar los objetivos de la organización. Es así como adoptan nuevas metodologías en el terreno de la comunicación, la gestión del desempeño, la capacitación y el relacionamiento (Contreras, et al. 2020).

En el teletrabajo también es clave una cultura basada en la confianza, sobre todo en esta pandemia que está presente la posibilidad de pérdidas económicas y de puestos de trabajo. Confianza mediante, y con ciertas incertidumbres atendidas, tanto trabajadores como empleadores pueden darle un valor significativo a esta modalidad de trabajo (Chávez Escobedo, et al, 2020).

En el contexto particular de la pandemia, los casos de comunicación calificados como exitosos por los expertos han sido los de aquellos líderes capaces de reconocer explícitamente el estado emocional general, por ejemplo, de miedo o preocupación, de redefinir, ajustar o reafirmar el propósito de la organización y de mantener comunicaciones frecuentes y empáticas (Dirani et al., 2020). Si bien el discurso y los mensajes de los líderes de la organización fueron claves para recuperarse del colapso de sentido que implicó esta crisis y plantear alternativas verosímiles en medio de un contexto de confusión y ansiedad (Weick, 1993; Yeomans y Bowman, 2021), algunas investigaciones señalan que el apoyo recibido de los compañeros de trabajo, a través de lo que podemos denominar canales de comunicación informal, fue fundamental para lidiar con la incertidumbre y el agotamiento emocional que aparejó esta crisis, incluso con un mayor impacto positivo que los canales de comunicación formal (Charoensukmongkol y Phungsoonthorn, 2020).

La tecnología, que es la herramienta que en definitiva hace posible mantener canales formales e informales de comunicación abiertos y activos durante la no presencialidad, y que permite que las organizaciones continúen operativas, también es la causante de mucho estrés. En pandemia, el tecnoestrés y los desórdenes psico-fisicos como ansiedad o insomnio, son los desórdenes más reportados en los trabajadores de diferentes países, especialmente en los países que optaron por confinamientos obligatorios como medida para evitar el avance de la pandemia (Zito et al., 2021)

Uruguay es un país que se caracteriza por su estabilidad política y económica. Sus instituciones públicas actúan con transparencia, son fuertes, extendidas y la población confía en ellas. Por otra parte, se encuentra en una zona geográfica libre de desastres naturales. Por ello las crisis sistémicas, de gran impacto, son muy excepcionales. Esto da condiciones muy buenas para los proyectos complejos y los de largo aliento, pero genera cierta debilidad a la hora de enfrentar una crisis ya que no hay experiencia ni aprendizaje acumulado al respecto.

Del procesamiento estadístico de los datos uruguayos del Latin American Communication Monitor, realizado especialmente para este estudio, se observa que el $73 \%$ de los profesionales encuestados que trabajan en empresas privadas consideró que la crisis generada por la pandemia del COVID-19 tuvo un impacto alto o total en el funcionamiento de su empresa. Con un $58 \%$ de estos encuestados participando en los comités de crisis de su empresa, y $35 \%$ con poder de decisión en ellos era pertinente investigar qué decisiones habían tomado, qué acciones habían implementado, qué prácticas habían desarrollado, en medio de esta crisis excepcional que sacudió la estabilidad uruguaya.

\section{Metodología}

El objetivo central de esta investigación, cualitativa y de carácter descriptivo, fue el relevamiento de los aspectos claves de la función de comunicación interna en las 
empresas uruguayas durante los primeros meses de desarrollo de la pandemia de COVID-19. El problema se enfocó en torno a las siguientes preguntas de investigación: ¿estaban preparadas las unidades de comunicación interna para esta crisis? ¿cambió la gestión de las unidades de comunicación interna con la pandemia? ¿cuáles fueron los desafíos? y ¿cuáles las proyecciones para comunicación interna?

Para la investigación se realizaron 15 entrevistas en profundidad de aproximadamente una hora de duración. La muestra, definida en forma teórica, fue de responsables de comunicación interna, del nivel más alto en su departamento, y que pertenecen a empresas privadas grandes, nacionales e internacionales, con instalaciones físicas en Uruguay. La muestra quedó compuesta finalmente con las siguientes características adicionales: (a) en cuanto a los profesionales, la casi totalidad de los entrevistados participa con decisión en reuniones del nivel más alto de su empresa, la casi totalidad tiene estudios universitarios de grado y postrado en comunicación; y (b) en cuanto a las empresas, la mitad cotiza en bolsa, y pertenecen a diversos sectores, agroindustria, industria, química, servicios financieros, servicios informáticos, retail y comercio electrónico.

En las entrevistas se aplicó una pauta semiestructurada con preguntas abiertas y preguntas con opciones para asistir al entrevistado en algunas preguntas. La pauta de entrevista relevó datos y percepciones de los entrevistados sobre los siguientes ejes temáticos: cambios en la función, la gestión y la aplicación de técnicas en las unidades de comunicación interna a partir de la emergencia sanitaria del COVID-19, preparación para atender la crisis a nivel general de empresa y a nivel de unidad de comunicación interna, experiencias anteriores a la pandemia en gestión de crisis, principales impactos de la emergencia sanitaria en la empresa, y formas de atender los impactos desde la comunicación, evaluación de la situación de comunicación interna a partir de la gestión realizada durante la crisis y perspectivas a futuro para la unidad de comunicación interna.

El trabajo de campo se realizó entre abril y mayo de 2021. Todas las entrevistas se realizaron por videollamada, lo que facilitó el contacto y la agenda de entrevistas. La realización de entrevistas por videollamada genera limitaciones al momento de captar percepciones sobre el entrevistado y su entorno laboral. Las expresiones no verbales que pudieran dar algún tipo de indicio sobre cómo se está respondiendo casi no tuvieron lugar por la limitación impuesta por la pantalla.

Luego de finalizado el trabajo de campo se realizó el análisis temático de contenido. En un primer momento se hizo la lectura completa de todas las entrevistas y se contrastó con los tópicos previstos en la pauta inicial. A partir de esa lectura se elaboró una malla temática de la que derivó los códigos de las unidades de análisis del texto de las entrevistas. La codificación integró categorías previstas en las pautas y categorías emergentes que surgieron durante las entrevistas. El corpus textual de las entrevistas fue analizado con estas categorías. Dado el número de entrevistas no fue necesario aplicar procesamiento informático.

Resultados preliminares de esta investigación se presentaron el 7 de octubre de 2021, en el XVIII Encuentro de la Federación Latinoamericana de Facultades de Comunicación (FELAFACS).

\section{Resultados}

Se presentan los principales resultados de las entrevistas, tematizados y con ejemplos textuales de los entrevistados.

\subsection{La preparación para la crisis de la pandemia}


Uruguay ha tenido muy pocas crisis de gran impacto en las últimas décadas y eso determina que la experiencia en gestión de comunicación de crisis entre los comunicadores entrevistados fuera casi nula. Por otra parte, ningún mapeo de conflictos potenciales realizado por los comunicadores con más experiencia en el área podía incluir un suceso con las características de esta pandemia. Es por esto que la expresión: sobre gestión de crisis se aprendió sobre la marchai, puede caracterizar la experiencia de casi todos los entrevistados.

En unas pocas empresas, que cuentan con mecanismos institucionales de gestión y distribución del conocimiento, se registró y ordenó la información en el correr de la pandemia, a fin de que sirva de aprendizaje: una vez que estas situaciones se superan, en la empresa se hace una reunión de "lecciones aprendidas". Con la gestión del conocimiento esas empresas pueden identificar qué acciones funcionaron bien, cuáles no, qué se realizó que no estaba planificado y puede ser bueno incorporar y qué planificado para gestionar una crisis no se llegó a implementar. A partir de esas reuniones en las empresas se definen los ajustes en los planes de gestión de crisis.

Para otros, que también registraron decisiones y acciones, resultó un aprendizaje personal destacado por tratarse de la primera crisis en la que su empresa incluía la comunicación interna como función esencial. Algunos pocos profesionales entrevistados no mostraron un aprendizaje transmisible de esta crisis, y la experiencia quedará restringida al conocimiento tácito personal o en los productos realizados.

Que se tratara de una crisis atendida en todo momento por las autoridades nacionales fue una característica particular. Las medidas gubernamentales, a diferencia de otros países, nunca incluyeron la cuarentena obligatoria, pero el poder ejecutivo tomó decisiones sobre el distanciamiento social, el uso de medidas sanitarias, los requisitos de aforo y exhortó al teletrabajo. Las decisiones tomadas en cada empresa estaban alineadas con lo dictaminado por el gobierno. Esto no les permitía flexibilidad en algunos aspectos, pero les dio libertad para resolver las situaciones que requerían trabajo presencial, por ejemplo, para no paralizar el funcionamiento de la empresa. Por otra parte, los entrevistados consideran las medidas del gobierno como un marco de seguridad que encuadró, sin mayores resistencias, las decisiones que se tomaron en las empresas tanto en lo sanitario como en lo laboral.

Los potenciales impactos del COVID-19 fueron observados de forma temprana en las empresas multinacionales con casas matrices en Europa o América del Norte, donde el COVID-19 golpeó antes que en América Latina y en Uruguay en particular. En estos casos recibieron ciertas advertencias: recibíamos extranjeros todas las semanas que venían del epicentro del brote. Fue así como empezamos a aplicar medidas prácticas de protección cuando en Uruguay nadie caminaba con tapabocas.

En la casi totalidad de las empresas a la que pertenecen los entrevistados, se creó un comité de crisis. Algunos estaban establecidos en sus manuales de crisis, con roles y funciones claramente detalladas. Otros, en cambio, fueron comités de crisis de forma ad hoc, muchas veces llamados comité COVID, con una integración que varió en el tiempo, de acuerdo con las necesidades que exigía la evolución de la pandemia.

Los entrevistados coinciden en que era esencial que la unidad de comunicación interna integrara el comité, y de hecho así lo hizo en todos los casos. En algunos, comunicación interna era solo una unidad ejecutora de las acciones que se decidían en el comité. En otros, fue una unidad medular y articuladora de la discusión y las decisiones en todos los aspectos referidos a la empresa.

Con los comités de crisis en funcionamiento las unidades de comunicación tuvieron una marcada vocación por informar a los trabajadores sobre el estado de situación de la empresa, desde el inicio de la pandemia en cuanto a lo sanitario, y más tarde también 
en los aspectos laborales. Esta información fue valorada como necesaria a fin de calmar la ansiedad y combatir la incertidumbre.

\subsection{Los desafíos del teletrabajo en pandemia}

La implementación abrupta del teletrabajo fue el desafío más importante para todos los entrevistados. En unas pocas empresas existía experiencia de teletrabajo anterior a la pandemia: en 2019 se comenzó a trabajar a distancia como un proyecto piloto. Nuestro negocio nos permitía hacer teletrabajo y teníamos antecedentes de otros países en los que está la empresa. Era algo que se veía venir, más allá del COVID, íbamos a tener teletrabajo.

Antes de la pandemia, la modalidad de trabajo a distancia generaba desconfianza en muchas empresas y en varios aspectos, por ejemplo, con respecto al potencial impacto en los resultados y el desempeño de la empresa, en cuanto a los requisitos para la implementación y sobre los ajustes legales requeridos para las condiciones laborales. La pandemia obligó su implementación y demostró, según responden varios entrevistados, que se puede aplicar sin que afecte la productividad, derribando los miedos y, además, con valoración positiva del personal. Un entrevistado, agrega: el trabajo desde la casa ya se venía pidiendo, y la respuesta de la empresa era que no estábamos tan convencidos... el 16 de marzo a nadie le importó si estábamos seguros o no, todos nos fuimos a casa y todos trabajamos.

La implementación del teletrabajo requirió la colaboración con otras áreas de la empresa para facilitar en muy poco tiempo, el acceso a computadoras, la conexión a internet, el equipamiento, mobiliario adecuado para el trabajo en casa. Para poder implementar esto muchos entrevistados realizaron un mapeo de las condiciones en las que trabajarían los colaboradores en sus casas, para contemplar las distintas realidades y requerimientos: se preguntó si tenían hijos, si tenían familiares a cargo o mayores de edad, si tenían medio de transporte, algún medio en el que trasladarse, si vivían cerca otro compañero, si creía que otro compañero los podía traer, si contaban con computadora, con internet, si estaban enfermos, detalló un entrevistado.

A continuación, se detallan los desafíos que el teletrabajo en pandemia impuso a las áreas de comunicación interna.

\subsubsection{Los ajustes en la gestión de la comunicación interna}

La pandemia y el teletrabajo modificó el funcionamiento de la comunicación interna en todas las empresas consultadas. Los entrevistados realizaron cambios en los contenidos, los planes, en las funciones de las unidades, y en lo operativo: las técnicas, canales y actividades de comunicación interna.

Para todos los entrevistados la comunicación sobre las medidas sanitarias fue el contenido priorizado durante los primeros días, con producción urgente de campañas y mensajes. En las empresas que no pudieron interrumpir el trabajo presencial, sectores industriales con operarios de plantas y comerciales con atención al público, la unidad de comunicación interna sumó como prioridad inicial comunicar la compleja logística de turnos, cuidando que los mensajes no generasen malestar entre los que iban a teletrabajo y los que permanecían en modalidad presencial y asegurando a los empleados que trabajar en la empresa era tan seguro como trabajar en casa.

Algunos entrevistados, con los planes de comunicación postergados, comentan: tuvimos que parar y ver dónde estaba la empresa, qué es lo que estaba pasando y de qué forma le aportábamos valor a la empresa, como un todo, en ese momento tan particular. Muchos mantuvieron las ideas fuerzas y los mensajes troncales de la unidad, y otros rehicieron por completo sus planes, entre ellos, las acciones que implican trabajo con la comunidad como sustentabilidad, responsabilidad social. 
Una de las funciones principales de los responsables de comunicación ante el abrupto paso al teletrabajo fue actuar como portavoz interno de sus autoridades. Casi todos afirman que la cantidad de comunicados oficiales se incrementó, y los comunicadores se involucraron junto a las autoridades, desde el inicio, en la creación del contenido de cada mensaje, en la decisión de qué decir, cómo y en qué momento.

Otro cambio singular en las funciones de comunicación interna fue el fortalecimiento de la función de asesoría en comunicación, muchas veces relegada a segundo plano. En algunos casos se formalizó un servicio de consultoría interna de comunicación, prestado desde la propia unidad para el resto de la empresa e, incluso, para otras filiales regionales. Esta función de asesoría resultó sorpresiva para varios y buscada para otros: cuando empezamos a asesorar, sabíamos que abríamos la puerta y se nos venían como avalancha. Y así fue, se acercaban a decir 'no sé cómo hacer para conectar con mi gente' y nosotros desde comunicación nos pusimos a trabajar para ayudarlos.

Con respecto a los cambios operativos, un entrevistado sintetiza la que fue primera preocupación en todas las áreas de comunicación interna de las empresas consultadas: elegir bien los canales de comunicación para esta situación, ver el grado de satisfacción con respecto a esos canales, [asegurarnos] de que las personas se sientan informadas de primera mano, que se enteren primero por nosotros y no por otro lado.

El servicio de mensajería, en particular WhatsApp, fue uno de los canales que, si bien se utilizaba con anterioridad en los niveles más altos de la empresa o para algunos objetivos y siempre con alcance acotado, fue legitimado ahora como un canal útil y necesario. Para la mayoría de las empresas el WhatsApp era considerado un canal personal, no corporativo. La situación de crisis llevó a que la inmediatez y masividad requerida posicionaran al WhatsApp como una alternativa óptima.

Con el WhatsApp llega el desafío de adaptar los contenidos: necesitábamos competir con grupos de WhatsApp con mensajes audiovisuales muy fuertes, entonces ya no basta con hacer una e-card o una comunicación con textos largos, porque la gente ya no los iba a leer o no les daba importancia, sostiene un entrevistado.

De todas maneras, este canal no consigue unanimidad. Algunos intentan mantener el número del teléfono móvil en la esfera personal privada $\mathrm{y}$, ante la inminencia de la necesidad de un servicio de mensajería instantánea, acuden a herramientas como Microsoft Teams, aunque es más utilizada para la conversación diaria y la asignación de tareas, que como medio de difusión masiva e instantánea.

La incertidumbre generada por la pandemia, el teletrabajo, y la necesidad de mensajes instantáneos y efectivos también llevó a revisar la utilidad del canal más expandido e interiorizado en comunicación interna: el correo electrónico. Vimos que teníamos que comunicar más, pero no tanto por mail, porque todos estábamos saturados y llega un punto en que se empieza a perder efectividad. Empezamos a necesitar una comunicación más ágil y dinámica, respondiendo rápido a las preguntas, siendo transparentes y contando lo que iba pasando, momento a momento, sostiene un entrevistado. En respuesta a esto algunas empresas se enfocaron en la realización de foros abiertos periódicos entre autoridades y personal.

Si bien en algunas empresas los foros abiertos virtuales sustituyeron a los presenciales que ya se hacían, para muchos son canales nuevos que aportan transparencia y cercanía a los trabajadores. Quienes antes integraban directorios que solo conversaban entre ellos, ahora se exponen frente a todos los colaboradores de la empresa y se abren a preguntas espontáneas con la responsabilidad de ofrecer una respuesta inmediata. Con el mismo esfuerzo que requiere organizar una reunión para veinte personas, con plataformas digitales como Zoom, Google Meet o Microsoft Teams se logra incluir a cientos de colaboradores en simultáneo. 
Las empresas multinacionales y las nacionales extendidas en gran parte del territorio, se vieron favorecidas por estas herramientas: todas las reuniones a través de herramientas digitales crecieron exponencialmente [en cuanto a la asistencia y participación]. Con las nuevas herramientas, nos dimos cuenta de que estas reuniones pueden ser para todo el mundo. En 2020 hicimos tres encuentros regionales con asistencia de autoridades de la empresa a nivel global. Allí estuvo invitada toda la región. Había mil personas conectadas al mismo tiempo, detalla un entrevistado. Los townhall [foros abiertos] que antes eran presenciales y trimestrales, pasaron a ser mensuales y virtuales. Esto cambió cómo nos comunicábamos y qué tipo de contenidos trabajábamos, además de la duración, agrega otro entrevistado.

En síntesis, el mayor desafío sobre los canales fue seleccionar uno efectivo para la comunicación inmediata, con alcance masivo, aplicable tanto a comunicación oficial como no formal y que permitiera la bidireccionalidad.

Otro cambio significativo fue convertir un sin número de actividades presenciales (reuniones, jornadas, eventos) en virtuales, y para ello, aprender a gestionar los límites de la modalidad a la vez que se aprovechan las oportunidades que abre. Esta es una de las prácticas que muchos esperan perdure una vez se levante la emergencia sanitaria.

\subsubsection{Los ajustes en la dirección de la comunicación interna}

A partir de la implementación del teletrabajo, el foco de los comunicadores, la orientación esencial de las unidades de comunicación interna se redirige al bienestar de las personas de las empresas.

\section{Mantener la cercanía}

Una vez que las empresas vieron el trabajo a distancia como una realidad y que la operativa no tenía interrupciones, los departamentos de comunicación interna identifican un desafío más importante: mantener la cercanía, con y entre el personal, en teletrabajo y en tiempos en que reinaba la incertidumbre por la situación sanitaria, la laboral y la económica. Dice un entrevistado: la pandemia, físicamente, nos alejó porque no nos escuchamos más en los pasillos, tuvimos la necesidad de estar juntos más allá de no tener un lugar para conversar, hay un tema de ánimo, de mantenerse juntos y más acompañados.

Surgen, en este tiempo, muchos programas focalizados a atender la distancia. Desde las clásicas capacitaciones o talleres, las charlas sobre nutrición, clases de yoga, propuestas lúdicas, concursos con premios: lanzamos un programa con actividades para conectar, para conocernos. No queríamos que, como ahora el trabajo era remoto, cada uno se quedara en la suya y se perdiera la cultura que teníamos. Hicimos eventos virtuales que fueron impresionantes, de colaboración, que incluía tener gente que no se conocía trabajando juntos para resolver un enigma para un juego, detalla uno de los entrevistados.

Algunos entrevistados ven la necesidad de que las personas en la empresa no pierdan las charlas informales para conversar de temas no laborales. Todas las semanas tenemos las kitchen meeting, que son esas charlas que se dan normalmente en la cocina, preparando el café. Ahora lo hacemos de manera virtual todos los jueves, 15 minutos, hacemos grupos aleatorios de cuatro o cinco personas y hablan entre ellos, cuenta un entrevistado.

\section{Monitorear la motivación y el bienestar}

La mayoría de los entrevistados realizan el monitoreo del clima y la motivación: todos los meses enviamos encuestas para tener el termómetro y saber qué atender y por dónde seguir. Estos monitoreos permitieron identificar muy rápidamente los problemas 
de motivación: en un momento se rompió la frontera entre el trabajo y la vida privada. La gente comenzó a estar sobrecargada, con video llamadas todo el tiempo que saturaban las agendas.

Antes estas alertas, algunos implementaron un servicio de escucha activa para sobrellevar la situación de aislamiento y distanciamiento entre los compañeros de trabajo. En algunos casos, incluso, los departamentos de comunicación integraron psicólogos para poner a disposición de quien lo necesita. El mensaje es que estoy cerca y entonces todos los días me importa comunicarme contigo que también estás trabajando. Tal vez estás con muchísima incertidumbre y yo estoy acá, comenta uno de los entrevistados para ilustrar el sentido del servicio. Otros fortalecen el trabajo en red entre compañeros de equipos, o refuerzan el ánimo con mensajes constantes de que entre todos podemos sobrellevarlo.

Los comunicadores en pandemia están adaptándose a algo desconocido y al mismo tiempo mantienen el contacto personal con los colaboradores, innovan todo el tiempo, buscan las mejores maneras de inspirar a la gente, de mantenerla motivada, refuerzan el sentido de pertenencia, animan ante el cansancio. Se preocupan por mantener los vínculos con las personas, entre sus compañeros y con la organización.

\section{$\underline{\text { Reforzar el liderazgo con confianza }}$}

El teletrabajo impidió el control tradicional basado en la supervisión directa. Los responsables de equipos rápidamente necesitaron transformar el control en autocontrol y para ello fue necesario trabajar el valor de la confianza, porque: del te estoy mirando y sé qué hacés, se pasó abruptamente a estás en tu casa y no sé nada. Los departamentos de comunicación trabajaron en talleres sobre estas nuevas responsabilidades, las nuevas formas de hacer el trabajo, la necesidad de que todos los líderes, de todos los niveles, estén atentos a su gente y, sobre todo, que se enfoquen en el desarrollo de la confianza como valor central de la cultura de la empresa.

Los gerentes de las empresas entendieron el sentido de cambiar su estilo de comunicación por uno más llano y cercano a sus colaboradores, con recorridas informales, con mensajes simples, con nuevas configuraciones de reuniones y encuentros. La orientación de las unidades de comunicación interna determinó que el contacto personal y el valor de la cercanía pase a tener un rol de mayor importancia para las principales autoridades de las empresas.

En este aspecto la mayoría de los entrevistados afirman la relevancia de la función de comunicación distribuida en la organización: la comunicación es más que la unidad de comunicación, la comunicación se lleva entre todos y principalmente las personas que lideran, dice un entrevistado, los líderes son los que tienen o deberían tener la capacidad de llevar la comunicación, para que las personas respondan a ellos, confíen y crean en su palabra, agrega otro entrevistado, los líderes son los dueños de la comunicación con su equipo, concluye un tercero. Por esto, la mayoría de los comunicadores entrevistados trabajan de igual a igual con los líderes formales de sus empresas, empoderándolos en la comunicación, para que sean ellos los comunicadores con su gente.

\subsection{El futuro para comunicación interna}

La comunicación interna ha sido la hermana menor de la comunicación externa, especialmente en las empresas de sectores que requieren mucha inversión en publicidad y marketing. Pero durante la pandemia la comunicación interna fue la protagonista.

El reconocimiento a los departamentos de comunicación interna es una constante. En algunos casos con reconocimientos formales de autoridades, en otros con el reconocimiento de los pares, incluso algunos se ven favorecidos con asignación de 
presupuesto para nuevos proyectos. En algunas empresas, además, las encuestas de clima muestran el reconocimiento del personal en su conjunto.

La comunicación interna adquirió un carácter aún más estratégico que el que tenía: hoy a nadie se le ocurre decir algo si no pasa por nosotros, porque confían que la mejor forma es consultar para comunicarnos mejor, dice un entrevistado. Tuvimos una buena recepción por los colaboradores y la gerencia a la hora de considerar a comunicación interna como aliado estratégico con el negocio, agrega otro.

Este carácter estratégico lleva a que los responsables de los departamentos, que antes no participaban en las decisiones estratégicas pasen a tener una legítima presencia en los espacios de toma de decisión.

Desde la visión más optimista de la pandemia y sus consecuencias, hay quien asegura que: esta pandemia fue lo mejor que le pudo pasar a las unidades de comunicación interna.

Entre los profesionales entrevistados hay unanimidad en que la virtualidad será un fenómeno ineludible en el futuro. En las empresas multinacionales este aspecto adquiere una valoración mayor porque se destaca la optimización del tiempo que significa poder concertar reuniones con autoridades en el extranjero, sin la necesidad de viajar y pasar días fuera del país.

De todos modos, los entrevistados marcan la importancia de no perder la presencialidad y afirman que a futuro surgirán nuevas reconfiguraciones del trabajo, cambiará el uso de los espacios, los grupos serán más dinámicos y flexibles en su integración. Pero, en su opinión, terminar con la presencialidad en forma total o casi total, podría debilitar la cultura de la organización. El recambio natural de trabajadores de una empresa, la iría convirtiendo en una organización en la que pocos se conozcan, no compartan espacios físicos y por lo tanto tengan dificultades en el relacionamiento, en el sentido de pertenencia y la cercanía entre los colaboradores.

Sobre el futuro inmediato, un aspecto que algunos de los entrevistados destacan es que se deberán ocupar del monitoreo de temas relacionados a la salud mental: se empezó a hablar con la excusa de la pandemia, pero se ha perdido el miedo a hablar de la salud mental, como algo que es inherente e igual que hacer un programa de deporte y ejercicio físico o alimentación saludable, resalta un entrevistado. Esta es, quizá, una perspectiva a futuro novedosa para la comunicación interna, porque pone entre sus funciones el bienestar integral de los colaboradores.

\section{Discusión}

Antes de la pandemia académicos y profesionales ya pronosticaban cambios disruptivos en el mundo del trabajo impulsados por la transformación digital. Desde finales del siglo pasado los empleados pueden trabajar desde cualquier lugar del mundo gracias a internet y los dispositivos tecnológicos, y esto genera la conformación de equipos dispersos geográficamente que desafían las estructuras y los liderazgos tradicionales (Gilson, et al., 2014; Dolan, et al., 2015).

Especialistas de diferentes campos investigan cómo impacta el trabajo remoto en el contrato psicológico, el compromiso y la satisfacción de los integrantes, y a su vez, cómo la cultura y el estilo de liderazgo de la organización impactan en la adopción y los resultados de ese entorno digital de trabajo (Tietze y Nadin, 2011; Köffer, 2015; Veeravalli y Vijayalakshmi, 2019; Vial, 2019). Los medios digitales, el cambio organizacional, las crisis, la confianza en los líderes, y la motivación y el compromiso son, desde hace al menos una década, los principales asuntos de los que se ocupa el área de comunicación interna (Verčič, et al., 2012). Por lo tanto, los issues que puso la 
pandemia entre los comunicadores uruguayos no son nuevos, pero su masividad e imposición abrupta los hace ineludibles y urgentes de atender.

Estudios recientes indican que el teletrabajo provocado por la pandemia se convirtió en una suerte de experimento global obligatorio que ha servido para anular algunos de los miedos más frecuentes relacionados a esta modalidad, y que en parte esto ha sido posible por la mejora de la digitalización y la innovación en tecnología (Dolce, et al. 2020). En el estudio se observa que los temores sobre el teletrabajo quedan rápidamente superados, y en lo que respecta a la función de comunicación interna es la oportunidad de ponerla como protagonista, en el centro de las funciones estratégicas de las empresas y con los valores esenciales de la comunicación con los públicos internos.

Investigaciones previas a la pandemia encontraron asociaciones positivas entre la adopción de esta modalidad de trabajo en casa, especialmente con el aumento de la productividad, la reducción del estrés, la reducción del impacto ambiental, la reducción de gastos personales de los trabajadores y el aumento del compromiso con la organización, en el contexto de pandemia, como vemos en el estudio. Pero el trabajo remoto durante la pandemia no fue una elección sino una obligación y los hijos de los trabajadores estaban también forzados a estar en los hogares todo el tiempo, el teletrabajo ocasionó una reducción del bienestar y un aumento del conflicto del balance vida laboral-familiar (Anderson y Kelliher, 2020). En las empresas estudiadas, el reconocimiento de estos riesgos del teletrabajo, atendidos rápidamente gracias a los monitoreos periódicos de clima, genera aprendizaje sobre cuáles podrán ser las mejores condiciones y prácticas para implementar el teletrabajo luego de pasada la pandemia.

Algunos estudios registraron que quienes comenzaron el teletrabajo de manera abrupta tuvieron una peor experiencia que aquellos que ya habían trabajado en esta modalidad mediante un acuerdo negociado con sus empleadores (Corrêa Gómez Cardim, 2021). Esta es una modalidad de trabajo que implica el ejercicio de la flexibilidad en tres aspectos no menores: el espacio, el tiempo y la definición de las tareas. Esta flexibilidad requiere una reconfiguración de los acuerdos de trabajo, las normas y condiciones laborales, procedimientos, estructuras de apoyo y, algo no menor, en el liderazgo (Aranda, 2021). En el estudio, se observa cómo los comunicadores pasan a tener un rol esencial como facilitadores de la comunicación de los líderes con sus equipos y en especial, en el fortalecimiento de la confianza como valor esencial de la cultura de sus empresas.

Entre las desventajas constatadas por la implementación del teletrabajo se observan, la percepción de la perdida de visibilidad del trabajador ante la organización dado el aislamiento, con el potencial perjuicio en su desarrollo, la pérdida del capital relacional de la red que le otorga la organización, además del surgimiento de conflictos en los equipos de trabajo por la hostilidad entre quienes realizan teletrabajo y quienes continúan de manera presencial (Tapasco-Alzate y Giraldo-García, 2020).

Como vemos en el estudio, los comunicadores de las empresas que requieren mantener la doble modalidad de trabajo se ocupan especialmente de las tensiones entre trabajadores. Los entrevistados agregan como desventaja que, pasar de forma completa al teletrabajo, podría tener impactos negativos en la cultura de la empresa. Seguramente la relación entre teletrabajo y cultura organizacional se profundizará en futuras investigaciones, y en cuanto a la profesión, será necesario pensar prácticas alternativas para construir y fortalecer la identificación y la pertenencia de los trabajadores con la empresa.

Un estudio de D'Almeida y Libaert citado por Castro-Martínez y Díaz-Morilla, (2020) muestra que previo a la pandemia, y ante el incremento de los casos de burn-out _agotamiento extremo por el sufrimiento laboral_ las organizaciones implementaron 
canales de comunicación ascendentes con el fin de dar un refuerzo en la comunicación que redunde en el bienestar de los colaboradores. Son ejemplos de estos canales los servicios de escucha implementados en algunas unidades de comunicación y los foros abiertos. A estos mecanismos de comunicación ascendente y bidireccional se agregan otro tipo de acciones como la creación de la figura del responsable de la gestión del bienestar y el diseño de espacios de convivencia y relajación, que como vemos, surge entre los entrevistados como una proyección para la función de comunicación interna.

Algunos estudios sobre el trabajo remoto ya venían señalando que, si bien puede ofrecer muchas ventajas, esta modalidad no resuelve per se el conflicto del balance entre el tiempo dedicado a familia o vida personal y al trabajo, sino que las condiciones que proporciona la organización: lo que habilita o no, la flexibilidad que permite o no, las prácticas de comunicación y trabajo, forman el factor crítico para superar ese conflicto (van der Lippe y Lippényi, 2020), por eso las prácticas que desarrollan los profesionales entrevistados son fundamentales para transitar por esta crisis y un valioso aprendizaje para el futuro del trabajo.

\section{Conclusión}

Con respecto a la primera pregunta que orientó esta investigación, la preparación de las unidades de comunicación interna para atender la crisis generada por la pandemia, observamos que las empresas y los profesionales de la comunicación no tenían experiencia previa en gestionar crisis dada la particularidad de que Uruguay es un país latinoamericano poco acostumbrado a lidiar con grandes crisis. Por ello esta experiencia ha sido inédita para todos los entrevistados. Incluso quienes tenían protocolos de comunicación de crisis establecidos, previo a la emergencia sanitaria, sintieron que aprendieron a gestionar la comunicación de crisis durante la pandemia.

En todas las empresas del estudio se instalaron comités de crisis, algunos con la comunicación interna como parte esencial de las decisiones y otros como unidad ejecutora de las decisiones tomadas en el comité. Se pudo observar situaciones diferentes en el aprendizaje organizacional que deriva de la pandemia. Al momento de realizar las entrevistas, las unidades de comunicación de empresas con mecanismos formales de gestión de conocimiento tenían sistematizado el aprendizaje. En otras empresas, sin experiencia en gestión del conocimiento, los profesionales desarrollaron un proceso de reflexión sistemática que les permitió generar aprendizaje. Pero, al momento de las entrevistas, unos pocos profesionales no habían convertido la experiencia de comunicación durante la crisis en aprendizaje.

Con respecto a la segunda y tercera pregunta del estudio, los cambios y desafíos en las unidades de comunicación interna con la pandemia, es evidente que ambos estuvieron centrados en atender los impactos de la adopción abrupta del teletrabajo.

El teletrabajo generó impacto en los empleados, especialmente aquellos que no están acostumbrados a teletrabajar, pero también en los líderes formales de las empresas, quienes no podían liderar de forma presencial y tuvieron que hacerlo a distancia. Para los comunicadores esto implicó desarrollar competencias en los líderes para comunicar de otra manera y lo hicieron a través de la función de asesoría interna, compartieron conocimiento y colaboraron a través de plataformas que para muchos fueron nuevas como Slack, Teams o Zoom, y en muchos casos empezaron a comunicarse a través de aplicaciones, no siempre utilizadas para la comunicación formal, como WhatsApp.

Medidas del gobierno nacional y el nivel de digitalización del país seguramente facilitaron la tarea en Uruguay. Pero el teletrabajo representó un desafío para las funciones centrales de la comunicación interna de todas las empresas: información de los asuntos centrales de la organización, alineación con la estrategia, asesoramiento y soporte a los liderazgos, compromiso de los integrantes, construcción de identidad y 
cultura. Todas las prácticas habituales en comunicación interna fueron impactadas por esta modalidad de trabajo.

Finalmente, con respecto a la pregunta sobre la proyección a futuro de la comunicación interna, el contexto de crisis le permitió probar a los profesionales uruguayos el valor que aporta la comunicación interna a sus empresas, para posicionarla como una de las funciones directivas de la organización, y que ocupe su lugar en las decisiones estratégicas con el rol esencial de asesoría interna.

Emerge además un llamado de atención entre los profesionales: la salud física y mental y el bienestar general de los integrantes de la empresa es un factor crítico para la continuidad operativa y no se puede descuidar. Este factor no solo será objeto de políticas públicas, como ya se comienza a discutir, sino que seguramente integrará la agenda interna de las empresas. Cómo apoyarse de la mejor manera en las tecnologías digitales para lograr el mejor desempeño, la mayor productividad y la más efectiva comunicación, sin descuidar, agobiar, alienar o enfermar a las personas, será sin duda un debate central en estos próximos años.

La investigación realizada tiene el valor de dar cuenta de aspectos de comunicación interna durante el momento más complejo de la pandemia en Uruguay, pero no recoge la experiencia de otros actores que interactúan con los comunicadores de estas empresas como empleados o personal directivo. Además, solo se restringe a un tipo de organizaciones en particular, las empresas privadas grandes y deja de lado organizaciones públicas o privadas sin fines de lucro, que pudieron experimentar impactos diferentes. Por otra parte, sería interesante explorar a futuro, en estas mismas empresas, si los cambios que generó la pandemia en las unidades de comunicación interna se sostienen en el tiempo.

\section{Referencias bibliográficas}

Agencia de Gobierno Electrónico y Sociedad de la Información y del Conocimiento (2020). Encuesta de Usos de Tecnologías de la Información y la Comunicación 2019. Montevideo: AGESIC. Recuperado de la página oficial del organismo: https://www.gub.uy/agencia-gobierno-electronico-sociedad-informacionconocimiento/datos-y-estadisticas/estadisticas/encuesta-uso-tecnologiasinformacion-comunicacion-2019

Álvarez-Nobell, A.; Molleda, J. C.; Moreno, A.; Athaydes, A.; Suárez-Monsalve, A. M. y Herrera, M. (2021). Comunicación estratégica y relaciones públicas en América Latina: crisis del COVID-19 en la gestión de comunicación, ciberseguridad, situación de las mujeres, retos éticos y nuevas competencias. Resultados de una encuesta en 20 países. Bruselas: EUPRERA

Anderson, D., y Kelliher, C. (2020). Enforced remote working and the work-life interface during lockdown. Gender in Management: An International Journal. Vo35, 677$683 \mathrm{http}: / /$ doi.org/10.1108/GM-07-2020-0224

Aranda, I. (2021). El teletrabajo, una nueva forma de entender la actividad laboral. Capital Humano, 100-104.

Arzuaga-Williams, M. (2020). Rol del comunicador organizacional en Uruguay: tareas y funciones. Profesional de la información, v. 29, n. 3. http://doi.org/10.3145/epi.2020.may.26

Arzuaga-Williams, M., Pepe, A.I., Izaguirre, M. y Rodríguez-Milhomens, G. (2021). Análisis de los resultados de Uruguay en el Latin American Communication 
Monitor, Montevideo: Universidad Católica del Uruguay. Recuperado de https://www.youtube.com/watch?v=U79Qgtz3wUc

Bojadjiev, M., \& Vaneva, M. (2021). The impact of COVID-19 crisis on a company's internal communication. Interdisciplinary Description of Complex Systems 19 (2), 239-256. http://doi.org/10.7906/indecs.19.2.5

Castro-Martínez, A., \& Díaz-Morilla, P. (2020). Comunicación interna y gestión de bienestar y felicidad en la empresa española. Profesional de la información, v. 29, n. 3, http://doi.org/10.3145/epi.2020.may.24

Contreras, F., Baykal, E., \& Abid, G. (2020). E-Leadership and Teleworking in Times of COVID-19 and Beyond: What We Know and Where Do We Go. Frontiers in Psychology. http://doi.org/10.3389/fpsyg.2020.590271

Corrêa Gomes Cardim, T., (2021). Crisis mundial de Covid-19 y teletrabajo: la nueva normalidad para las relaciones laborales. Revista Internacional y Comparada de Relaciones Laborales y Derecho del Empleo, Vol. 9, №. 1, pp. 352-376

Chávez Escobedo, J.M., Martínez Macías, J.G., \& Martínez González, J. F. (2020). Tecnología en el Trabajo. Revista Daena (International Journal of Good Conscience), 15(1), 256-263

Charoensukmongkol, P., y Phungsoonthorn, T. (2020). The interaction effect of crisis communication and social support on the emotional exhaustion of university employees during the COVID-19 crisis. International Journal of Business Communication, 1-18. Recuperado de https://journals.sagepub.com/doi/full/10.1177/2329488420953188

Dirani, K. M., Abadi, M., Alizadeh, A., Barhate, B., Garza, R. C., Gunasekara, N., Ibrahim,G., y Majzun, Z. (2020). Leadership competencies and the essential role of human resource development intimes of crisis: a response to Covid-19 pandemic. Human Resource Development International, 23(4), 380-394. http://doi.org/10.1080/13678868.2020.1780078

Dolan, S., Makarevich, A., y Kawamura, K. (2015). Are you and your company prepared for the future of work in tomorrowland? European Business Review, 4-12.

Dolce, V., Vayre, E., Molino, M., \& Ghislieri, C. (2020). Far Away, So Close? The Role of Destructive Leadership in the Job Demands-Resources and Recovery Model in Emergency Telework. Social Sciences. https://doi.org/10.3390/socsci9110196

Gilson, L., Maynard, T., Jones Young, N., Vartiainen, M., y Hakonen, M. (2014). Virtual Teams Research: 10 Years, 10 Themes, and 10 Opportunities. Journal of $\begin{array}{llll}\text { Management } & \text { Vol. } & \text { No.5,1313-1337. }\end{array}$ http://doi.org/10.1177/0149206314559946

Köffer, S. (2015). Designing the digital workplace of the future-what scholars recommend to practitioners. Thirty Sixth International Conference on Information Systems

Morelos-Gómez, J., \& Lora-Guzmán, H. S. (2021). Análisis del teletrabajo en las agencias de viajes turísticas en la ciudad de Cartagena-Colombia. Saber, Ciencia y Libertad, 16(1), 116-127. http://doi.org/10.18041/23823240/saber.2021v16n1.7522

Putnam, L. L. y Nicotera, A. M. (Eds.). (2009). Building theories of organization: The constitutive role of communication: Routledge.

Tapasco-Alzate, O. \& Giraldo-García, J. (2020). Teletrabajo: aspectos críticos para su implementación desde la perspectiva de los directivos 16th LACCEI International 
Multi-Conference for Engineering, Education, and Technology: Lima, Perú http://doi.org/10.18687/LACCEI2018.1.1.221

Tietze, S., \& Nadin, S. (2011). The psychological contract and the transition from officebased to home-based work. Human Resource Management Journal, 21(3), 318334. http://doi.org/10.1111/.j.1748-8583.2010.00137.x

van der Lippe, T., y Lippényi, Z. (2020). Beyond formal access: Organizational context, working from home, and work-family conflict of men and women in European workplaces. Social Indicators Research, 151(2), 383-402. http://doi.org/10.1007/s11205-018-1993-1

Vattuone, X. R. (2021). Teletrabajo y capitalismo de vigilancia. Telos, 23(1), http://doi.org/10.36390/telos231.14

Veeravalli, S., y Vijayalakshmi, V. (2019). A morphological review of enterprise social media literature. Journal of Organizational Computing and Electronic Commerce, 29(2), 139-162. http://doi.org/10.1080/10919392.2019.1583456

Verčič, A. T., Verčič, D., y Sriramesh, K. (2012). Internal communication: Definition, parameters, and the future. Public relations review, 38(2), 223-230. http://doi.org/10.1016/i.pubrev.2011.12.019

Vial, G. (2019). Understanding digital transformation: a review and a research agenda. Journal of Strategic Information Systems 28, 118-144. http://doi.org/10.1016/j.jsis.2019.01.003

Weick, K. E. (1993). "The collapse of sensemaking in organisations: the Mann Gulch disaster", Administrative Science Quarterly, Vol. 38, pp.628-652 http://doi.org/10.2307/2393339

Yeomans, L., y Bowman, S. (2021). Internal crisis communication and the social construction of emotion: university leaders' sensegiving discourse during the Covid-19 pandemic. Journal of Communication Management, 25 (3). 196-213. http://doi.org/10.1108/JCOM-11-2020-0130

Xifra, J. (2020). Comunicación corporativa, relaciones públicas y gestión del riesgo reputacional en tiempos del Covid-19. Profesional De La Información, 29(2). doi.org/10.3145/epi.2020.mar.20

Zito, M., Ingusci, E., Cortese, C.G., Giancaspro, M.L., Manuti, A., Molino, M., Signore, F., y Russo, V. (2021). Does the end justify the means? The role of organizational communication among work-from-home employees during the COVID-19 Pandemic. Int. J. Environ. Res. Public Health, 18, 3933. http://doi.org/10.3390/ijerph18083933

\footnotetext{
' Las respuestas textuales de los entrevistados están expresadas en cursiva.
} 